\title{
A computational approach to implicit entities and events in text and discourse
}

\author{
Rodolfo Delmonte
}

Received: 14 October 2009 / Accepted: 15 October 2009

(C) Springer Science+Business Media, LLC 2009

\begin{abstract}
In this paper we will focus on the notion of "implicit" or lexically unexpressed linguistic elements that are nonetheless necessary for a complete semantic interpretation of a text. We refer to "entities" and "events" because the recovery of the implicit material may affect all the modules of a system for semantic processing, from the grammatically guided components to the inferential and reasoning ones. Reference to the system GETARUNS offers one possible implementation of the algorithms and procedures needed to cope with the problem and enables us to deal with all the spectrum of phenomena. The paper will address at first the following three types of "implicit" entities and events:

- the grammatical ones, as suggested by a linguistic theories like LFG or similar generative theories;

- the semantic ones suggested in the FrameNet project, i.e. CNI, DNI, INI;

- the pragmatic ones: here we will present a theory and an implementation for the recovery of implicit entities and events of (non-) standard implicatures.
\end{abstract}

In particular we will show how the use of commonsense knowledge may fruitfully contribute to find relevant implied meanings. Last Implicit Entity only touched on, though for lack of space, is the Subject of Point of View, which is computed by Semantic Informational Structure and contributes the intended entity from whose point of view a given subjective statement is expressed.

\footnotetext{
R. Delmonte ( $₫)$

Ca' Bembo, Dorsoduro 1075, Università "Ca Foscari”,

30123 Venezia, Italy

e-mail:delmont@unive.it

url: http//project.cgm.unive.it
}

Keywords Deep linguistic processing - Semantic interpretation - Inferencing - Implications and implicatures . Common sense reasoning

\section{Introduction}

In this paper we will focus on the notion of "implicit" or lexically unexpressed linguistic elements that are nonetheless necessary for a complete semantic interpretation of a text. We referred to "entities" and "events" because the recovery of the implicit material may affect all the modules of a system for semantic processing, from the grammatically guided components to the inferential and reasoning ones.

The main difference existing between shallow syntactic methods mainly represented by the dependence parsing framework, and the linguistically oriented Semantic Text Processing systems can be gaged by the ability to compute Implicit Entities and Events (hence IEEs) which is viable only in the latter but not in the former type of systems. Shallow and dependency oriented approaches usually deal only with the actual words lexically expressed in a text. On the contrary, deep approaches allow the system to delve into the lexically and linguistically motivated IEEs by positing the existence of empty categories. Empty categories are placeholders for a number of different entities mostly recovered by means of chains of indices. They also respond to grammatical principles of various kinds, such as the universal need that any predicate must have a SUBJect — but more on these topics in the first section below.

Former systems make use of shallow lexica, which can be constituted by semantic relations as the ones present in WordNet which require only word level semantic relation matching. Using complex subcategorized lexica like COMLEX or NOMLEX (see website), FrameNet (Baker et al. 
1998), and other similar computational lexica, requires a different approach to the task of semantic processing which may account for IEEs.

Systems using these lexica must be able to distinguish Arguments from Adjuncts and to build appropriate representations for Predicate Argument Structures: this in turn requires clause level segmentation. These latter structures become then the input to the semantic interpreter that can apply principles of grammatical, lexical and semantic wellformedness to the analysis. It is just in this phase that the presence of IEEs can be detected by the system and an adequate semantic representation can be built. IEEs can be classified according to the following linguistically motivated subdivision:

a. grammatically motivated IEEs

$\circ$ as a subtype, IEEs identified by pronominal binding

b. semantically motivated IEEs

$\circ$ as a subtype, IEEs identified by anaphoric binding

c. pragmatically motivated IEEs

o semantically inferred IEEs

d. discourse motivated IEEs

- Centering Main Topic IEEs

We will now give examples of the four types and comment on their status in a theoretical and computational framework. The presentation will use LFG (Bresnan 2000) as linguistic theory and FrameNet as semantic lexical theory; semantic representations are inspired by Situational Semantics.

In the Penn Treebank II there are approximately 65,000 empty categories, the majority of which are constituted by traces - in a Chomskian sense (our grammatical IEEs)—of moved material. Empty elements constituted by unexpressed Subjects of untensed clauses are some 27,800. Then there is some 580 elliptical empty elements. If we consider that the PTB contains 93,539 sentences we see that there is almost one such empty element per sentence. Since 38,133 are SBAR clauses, they each contain one empty coindexed element. Hence, computing implicit elements is an important component of any semantically viable text analysis component.

\section{The system GETARUNS}

We can think of the system as being subdivided into two main meta-modules or levels: Low Level System, containing all modules that operate at Sentence Level; High Level System, containing all the modules that operate at Discourse and Text Level by updating the Discourse Model. The system is a top-down depth-first DCG-based parser written in Prolog Horn Clauses, which uses a strong deterministic policy by means of a look-ahead mechanism with a WFST to help recovery when failure is unavoidable due to strong attachment ambiguity.
SYSTEM ARCHITECTURE I

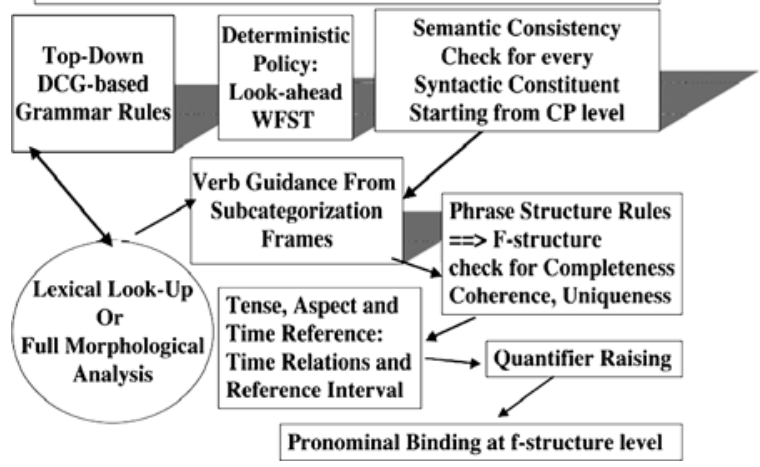

Fig. 1 Low level GETARUNS

It is divided up into a pipeline of sequential but independent modules which realize the subdivision of a parsing scheme as proposed in LFG theory where a c-structure is built before the f-structure can be projected by unification into a DAG (Direct Acyclic Graph). In this sense we try to apply in a given sequence phrase-structure rules as they are ordered in the grammar: whenever a syntactic constituent is successfully built, it is checked for semantic consistency. Whenever the governing predicate expects obligatory arguments to be lexically realized they will be searched and checked for uniqueness and coherence as LFG grammaticality principles require.

Syntactic and semantic information is accessed and used as soon as possible: in particular, both categorial and subcategorization information attached to predicates in the lexicon is extracted as soon as the main predicate is processed, be it adjective, noun or verb, and is used to subsequently restrict the number of possible structures to be built. Adjuncts are computed by semantic compatibility tests on the basis of selectional restrictions of main predicates and adjuncts heads.

The output of grammatical modules is then fed onto the Binding Module (BM) which activates an algorithm for anaphoric binding. Antecedents for pronouns are ranked according to grammatical function, semantic role, inherent features and their position at f-structure. Eventually, this information is added into the original f-structure graph and then passed on to the Discourse Module (DM).

The grammar is equipped with a core lexicon containing most frequent 10,000 fully specified inflected word forms where each entry is followed by its lemma and a list of morphological features, organised in the form of attribute-value pairs. In addition, there are all the lexical forms provided by a fully revised version of COMLEX, LCS, FrameNet, PropNet and VerbNet (see websites). Their grammatical verbal syntactic codes have then been adapted to our formalism and are used to generate a subcategorization scheme with an aspectual and semantic class associated to it-however no restrictions can reasonably be formulated on arguments 
of predicates. Semantic inherent features for Out of Vocabulary Words, be they nouns, verbs, adjectives or adverbs, are provided by a fully revised version of WordNet (Fellbaum 1998)—plus EuroWordnet, with a number of additions coming from computer, economics, and advertising semantic fields-in which we have used 75 semantic classes similar to those provided by CoreLex.

When each sentence is parsed, tense aspect and temporal adjuncts are accessed to build the basic temporal interpretation to be used by the temporal reasoner. Eventually two important modules are fired: Quantifier Raising and Pronominal Binding. QR is computed on f-structure which is represented internally as a DAG. It may introduce a pair of functional components: an operator where the quantifier can be raised, and a pool containing the associated variable where the quantifier is actually placed in the f-structure representation. This information may then be used by the successive higher system to inspect quantifier scope. Pronominal binding is carried out at sentence level. DAGs will be searched for binding domains and antecedents matched to the pronouns (if any) to produce a list of possible bindings. The best candidates will then be chosen.

\subsection{The upper module}

GETARUNS has a highly sophisticated linguistically based semantic module which is used to build up the Discourse Model. Semantic processing is strongly modularized and distributed amongst a number of different submodules which take care of Spatio-Temporal Reasoning, Discourse Level Anaphora Resolution, and other subsidiary processes such as Topic Hierarchy which cooperate to find the most probable antecedent of coreferring and cospecifying referential expressions when creating semantic individuals. These are then asserted in the Discourse Model (hence the DM), which is then the sole knowledge representation used to solve nominal coreference. The system uses two resolution submodules which work in a sequence: they constitute independent modules and allow no backtracking. The first one is fired whenever a free sentence external pronoun is spotted; the second one takes the results of the first submodule and checks for nominal anaphora. They have access to all data structures simultaneously and pass the resolved pair, anaphor-antecedent to the following modules. Semantic Mapping is performed in two steps: at first a Logical Form is produced which is a structural mapping from DAGs onto unscoped well-formed formulas. These are then turned into situational semantics informational units, infons which may become facts and/or sit(uation)s. Each unit has a relation, a list of arguments which in our case receive their semantic roles from lower processing - a polarity, a temporal and a spatial location index. Inferences can be drawn on the facts repository as will be discussed below.
SYSTEM ARCHITECTURE II

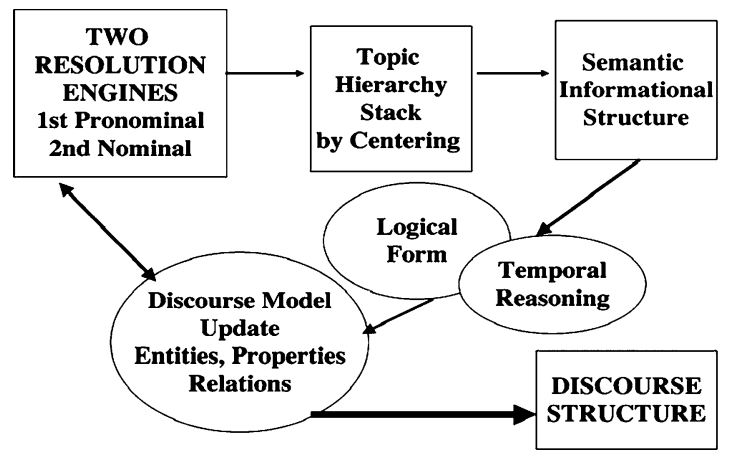

Fig. 2 High level modules of GETARUNS

\section{Grammatically motivated IEEs or GIIEs}

According to LFG theory as proposed by [1] grammatical IEEs may be classified into three types:

- Lexical Control

- Syntactic Control

- Structural Control

In order to activate control mechanisms, two procedures need to be implemented in the parser: an indexing function that distinguishes phrase structures from one another; a coindexing function and a corresponding chain climbing function to recover the semantic identity or the head of the controller.

\subsection{Lexical control IEEs}

Lexical control IEEs are constituted by empty categories that ensue from the presence of a phrase structure which has been computed as argument of the governing predicate and needs the presence of a SUBJect. An empty category is created which is then coindexed with the lexical control argument. Relevant examples are (1), (2) and (3) below:

(1) Mary asked John to buy a book

(2) John considers Mary an important ally

(3) Tom is a republican.

In (1) the predicate BUY is associated to an empty SUBJect which is coindexed with the controller JOHN in force of the existence of a lexical rule that selects a controller between a hierarchy of Grammatical Functions associated to lexically expressed arguments of the governing predicate, in this case ASK. The hierarchy is the Default Rule of Lexical Control and establishes the following order:

\section{(3.1) OBJ $2<$ OBJ $<$ SUBJ}

and simply says that the lexical controller is an OBJ2 (if present), an OBJ (if present), otherwise a SUBJ. In (2) the predicative NP "an important ally" is controlled lexically by 
the OBJect Mary in force of the same Default Rule, where the argument receives a semantic role from the lexical controller as well. Same situation with (3) where however the SUBJect is the controller of the predicative NP "a republican".

\subsection{Syntactically controlled IEEs}

Syntactically controlled IEEs refer to what are also called long distance dependencies. These constructions concern two types of clauses: relative clauses and interrogative clauses. These are too well known by computational linguists to require a presentation. We just include two examples to complete the description:

(4) Tom wanted the book that Mary bought.

(5) Which book did Mary buy?

In both examples the predicate BUY needs the existence of an empty category which is then filled by or coindexed with the syntactic controller, the BOOK. This may be achieved by different procedures depending on each linguistic theory, but the final result is always the same: a chain between two structures one of which has a control index added by the grammar.

\subsection{Structurally controlled IEEs}

Structurally controlled IEEs are those SUBJects that come into existence whenever there is a predicative ADJunct in a certain structural configuration or there is a dislocated structure. We include here below the relevant examples:

(6) John went to the see the movie drunk.

(6.1) John accompanied Mary to the movie naked.

(6.2) Drunk as usual John went to see the movie.

(6.3) Naked as usual John took Mary to the movie.

(7) The company has sold its assets to collect funds.

(7.1) These assets have been sold to collect funds.

**(7.2) These assets sell well to collect funds.

(8) After reading the letter Mary rushed to the school.

(8.1) Mary met John after finishing school.

(8.2) Reading books is important.

(8.2.1) Reading books is important for John.

(9) Ski John loves!

(9.1) At the corner was standing a young girl.

These examples do not exhaust all possible cases of structurally relevant IEEs. We have intentionally omitted cases of so-called "parasitic gaps" which we consider too rare in real texts to be taken into consideration. We also omitted the case called OBJect intransitivization, which will be discussed in the following section.

Example (6) is a case of an adjectival ADJunct which has the SUBJect as controller. This may be due to the unsuitability of MOVIE as controller of DRUNK. If we look at example (6.1), possible controllers are both Mary and John for
NAKED. So the OBJect Mary is taken. However, according to the position of the ADJunct control may pass to the SUBJect John. In other words, structural control does not answer only to grammatical criteria, but also to semantic criteria and finally to positional ones.

Example (7) is an interesting case where we see that the controller may also be omitted and in that case it needs to be restored from previous discourse. We deal with such cases below. Notice here the important fact represented by (7.2) where we included an ungrammatical case-a sentence that will not be found in real texts. Whenever the AGENT is not lexically expressed nor can be posited by grammatical principles no control may ensue. Crucially then, in order for a control structure like the RESULTATIVE infinitive to be expressed in a sentence, some controller needs also to be there. Then we have cases represented by examples under (8) which are all gerundives. As it seems, the controller is always the SUBJect disregarding its position. The copulative construction in (8.2) introduces another type of control, the one called ARBITRARY control. As can be noticed, (8.2.1) is no longer a case of Arbitrary control because of the presence of a BENEFICIARY "for John" who becomes the controller. Final cases are those constituted by so-called inverted focus structure — example (9)—and locative inversion in (9.1). These cases do not require the insertion of an empty category but a shallow parser is usually unable to cope with them appropriately (Delmonte 2007-2009). Deep processing will impose an appropriate argument structure by means of selectional restrictions, but also compute as SUBJect the inverted NP in the locative construction.

In Figs. 3 and 4 we show where and how Functional Mapping into Semantic Roles and Semantic markers is carried out in our system. This is also where Grammaticality Principles are checked for Completeness, Coherence and Uniqueness. In practice, what happens is that Control is checked at three levels, Syntactic, Functional and Lexical. Adjuncts are checked to see whether they precede or follow the main verb. Coindexing takes place only with Predicative or Open Functions.

\section{FUNCTIONAL MAPPING}

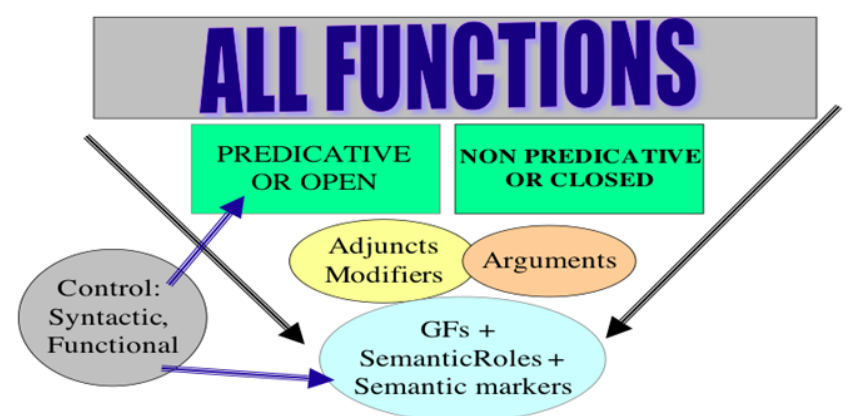

Fig. 3 Functional mapping with GETARUNS 


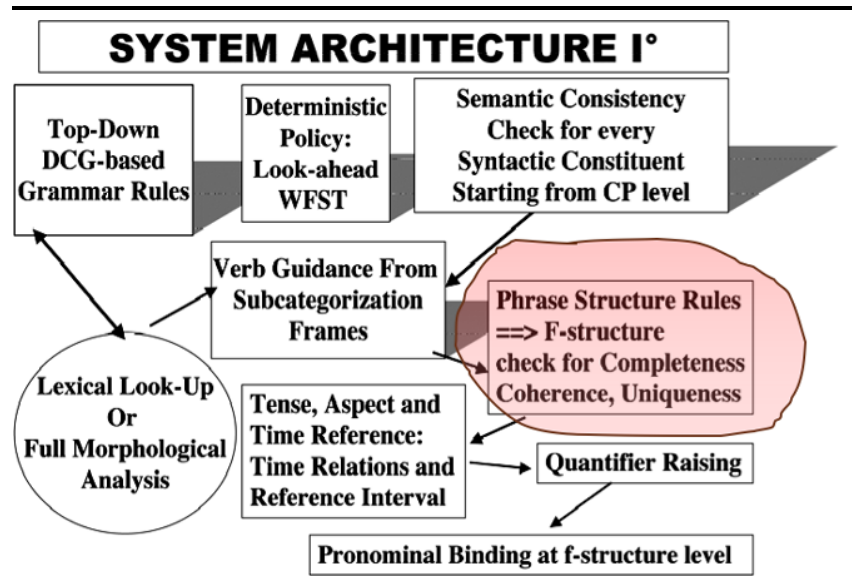

Fig. 4 Computational location of GIIEs

\section{Semantically motivated IEEs}

In this section we discuss cases of lexical-semantic IEEs which are also discussed in the FrameNet project, which is a theory of lexical representation and is based on its underlying linguistic theory, i.e. Constructional Grammar. Unlike our approach which is mainly computational, this project is descriptive and wrongly conflates cases of lexical semantics IEEs with cases of grammatical IEEs in the same typology. Starting from CNI, this class of implicit entities concerns structurally omitted constituents as can be gathered from the definition given in their Manual (ibid. p. 54).

Under the term CNI we find three types of IEEs conflated under the same definition, some of which have already been discussed above. Computationally speaking, these types require totally different tools and procedures to be activated. They may be redefined as follows:

\subsection{Pronominal binding IEEs or PIIEs}

(Big-)PRO cases as found in independent or Adjunct infinitives, participials and gerunds, i.e. in clauses with an untensed verb.

These cases have already been presented above. The PRO SUBJect inherits lexical properties associated to the subcategorization frame and may thus be pronominally bound to a structural controller, if any exists. Otherwise, the PRO is computed as generic or arbitrary: as a result, PRO cannot possibly be computed as external pronouns that can corefer in the discourse.

Finally, for these CNI to be computed, their presence is posited by the Interpretation Component of the system, which recovers Predicate Argument Structures or PASs by applying grammatical completeness and other principles to the output of the parser-in our case to c-structure. The output of the Interpretation Component are f-structures, i.e. semantically complete PASs. Big-PROs will then be bound by

\section{SYSTEM ARCHITECTURE I ${ }^{\circ}$}

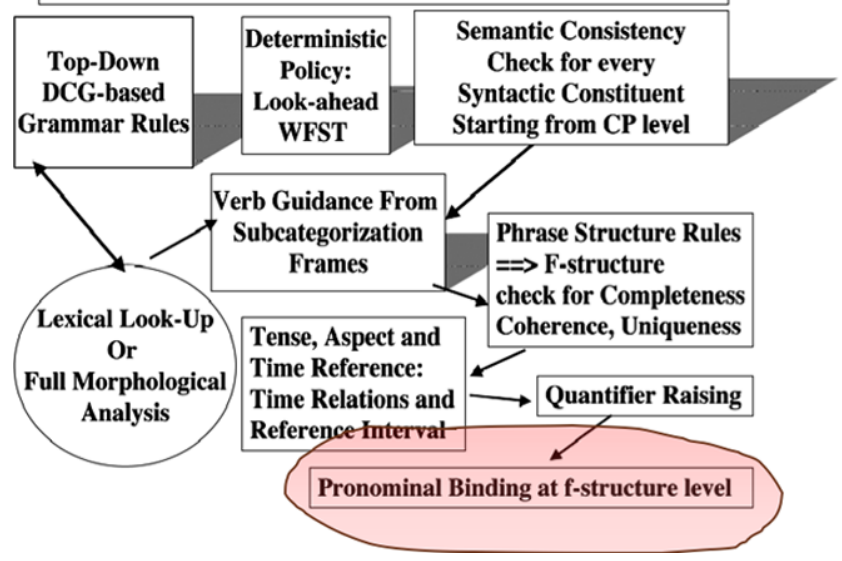

Fig. 5 Computational location of PIIEs

the Pronominal Binding component of the system, which works only at sentence level, using structural information and principles of the grammar.

\subsection{Anaphoric binding cases}

At first we have little-PRO cases for those languagesRomance but not only-that allow a SUBJect to be left lexically unexpressed in clauses with a tensed verb. The pronoun is added by the Interpretation Component as above and may be bound at sentence level. In addition, and differently from big-PRO it may become an external pronoun, which is then bound at discourse level. In this case, discourse level processing components like Topic Hierarchy and Centering - which will be presented below-will contribute to find the appropriate antecedent. This case includes IMPERATIVE mood sentences which require the SUBJect to be left unexpressed; COORDINATE structures with omitted understood SUBJect as in the example, "John went out and pro met Mary" which we comment on below, where the omitted SUBJect must be copied from the previous clause by the grammar embodied by the parsing module, rather than by interpretation or by lexically related principles. There is always the need to have a SUBJect expressed with all verb predicates.

In either case-i.e. in the case of sentence level pronominal binding or anaphoric discourse level binding - a decision must be taken by the Pronominal Binding module at sentence level.

\subsection{Ellipsis: on the edge between syntax and semantics}

We take all cases of ellipsis to be collapsed in a single computational action: copy of the elliptical material in the place where it is missing. This can only be done once the complete utterance that precedes the elliptical one has been fully 
parsed. Also it is important to bear in mind the fact that this process can be spotted only in case there is no ambiguity, as shown in the examples below:

(10) John went out and [John] met Mary

- SUBJECT null in coordination

(11) John often kisses Mary, and Bill does/will [kiss Mary], too.

$$
\text { - VP ellipsis }
$$

(12) John carefully counted the money, and Bill did/will [carefully count the money], too.

- VP ellipsis and Adverb ellipsis

(13) Harry lives in Boston and Mike [lives] in New York

$$
\text { - Gapping }
$$

(14) Susie wants to buy a car and my brother [wants [to buy]] a bike.

- LD Gapping

(15) Venice is the city where I live and [where] I work

\section{- Forward Conjunction Reduction}

(16) Some have served mussels to Sue while others have [served] swordfish

- PseudoGapping

(17) He takes and never gives back.

- Indefinite Null Instantiation (INI)

(18) What? Who?

- Sluices

As can be easily noticed, the only case in which ambiguity may constitute a problem is (16), PseudoGapping, which is a case of Auxiliary ellipsis, and English auxiliaries are ambiguous between lexical and non lexical usage.

In order to activate procedures for Elliptical sentence reconstruction, there must be a failure in the system. This is forced by the need to satisfy local grammatical constraints.

\subsubsection{Discourse model cases}

Here we have cases of Omitted Agent of passive sentences already discussed above, which we treat as we do cases of OBJect intransitivization, i.e. by adding a dummy existential quantifier. The Agent of passive sentences will then be identified by the semantic processing module which will look for a similar governing predicate in the context, or previous stretch of discourse. When the predicate is found the argument will be identified and the current existential bound to it in the Discourse Model. We will find some such cases in fully documented cases we discuss below.

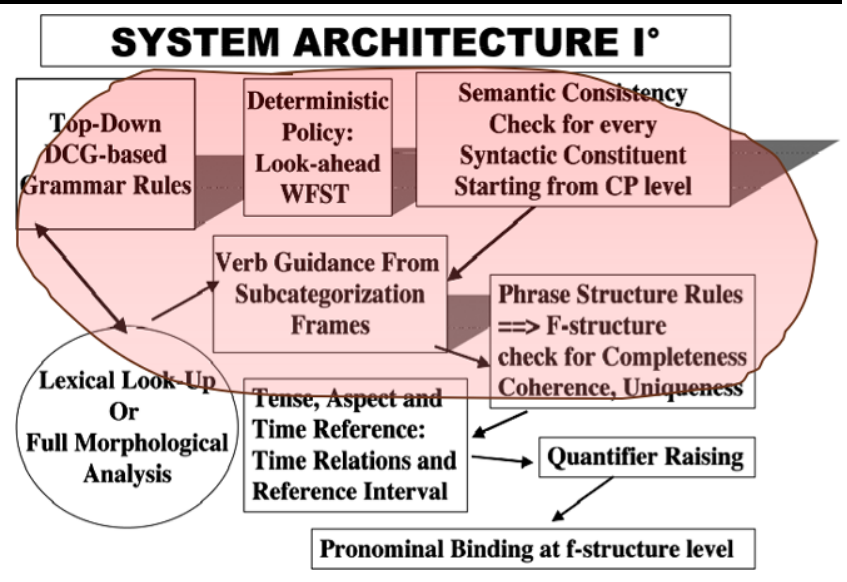

Fig. 6 Computational location of EIIEs

\subsubsection{Semantic coreference cases}

There is no need to specify a dummy (big)-PRO, little pro or existential quantifier in these cases because the missing element is an Adjunct and not an Argument as was the case with the examples discussed above. So the only way to recover the identity of the lexical entities coreferred by the optional adjuncts "evoked" by these structures is to search in the context, or in the previous stretch of discourse-the Discourse Model-for a similar semantic relation. When an identical predicate is found, the arguments are recovered and their semantic identifiers used to complete the extended PAS for the current predicate.

\subsubsection{Other discourse model cases}

We postulate a semantic treatment of empty deleted OBJect for those transitive verbs that allow it in the lexicon. The solution to the problem lies in the lexical nature of the phenomenon of OBJect intransitivation, which must be marked for Intransitivization, i.e. these are transitive verbs that may become Intransitives. Seen that transitive verbs constitute the great majority of all verbs in any language, and the ones allowing intransitivization is a small subset, they shall have to be marked so. The empty OBJect can then be added to the extended PAS by the semantic component. Similar cases are constituted by the deletion of OBJ 2 in ditransitive verbs, as also shown by example (17) above. So, we prefer to consider the OBJ2 as an existential that needs to be recovered, and leave the OBJ unexpressed.

\section{Semantically and/or pragmatically motivated IEEs?}

In this section we will introduce documented examples of various types of semantic and pragmatic IEEs. We shall use SEMANTIC to characterize all those cases of IEEs which 
involve lexically expressed or grammatically related phenomena. On the contrary, PRAGMATIC will be used to define extralinguistic IEEs, i.e. all those cases of IEEs which are implied, unexpressed and cannot be defined on the basis of grammatical or semantic rules, which require the use of knowledge of the world and preferential strategies to disambiguate. To present the output of semantic representations we shall use Situation Semantics inspired representations which are FACTS organized as follows:

\section{A FACT is an \\ Infon(Index, \\ Relation(Property), \\ List of Arguments-with Semantic Roles, \\ Polarity -1 affirmative, 0 negation, \\ Temporal Location Index, \\ Spatial Location Index)}

The representations are computed on the run and then collected at the end of the parsing of the whole text, this time by collapsing all events, properties and attributes assigned automatically to each entity in the text.

\subsection{Examples of semantically motivated IEEs}

Text 1 . The three friends went all outdoors. As they were walking in the garden, John said to himself, "Sara will marry that man", without any resentment. Richard would marry Sara. He felt strongly about that.

Triggering LD: Deictic pronoun THAT coreferring with previous adjacent event

Computational Strategy: The adjacent EVENT is built into a semantically complete PAS, with the ontological status of generic ENTity, and is coindexed with THAT-more on this text below.

Text 2. John gave Mary a rose. She took it and put it in her hair. She knew that she had been given a present, something precious.

Triggering LD: SUBJECT Ellipsis; Implicit Agent in Passivized structure

Computational Strategy: Indefinite NP to cospecify a property by recovering the identical relation in the DMtriggered by PlusPerfect

John gave Mary a rose.

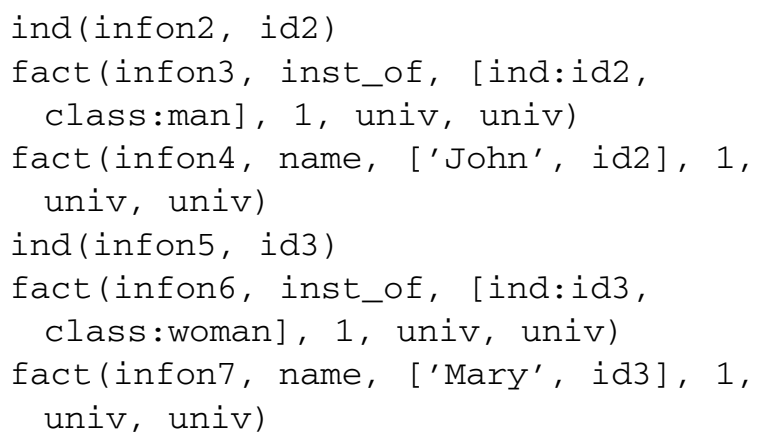

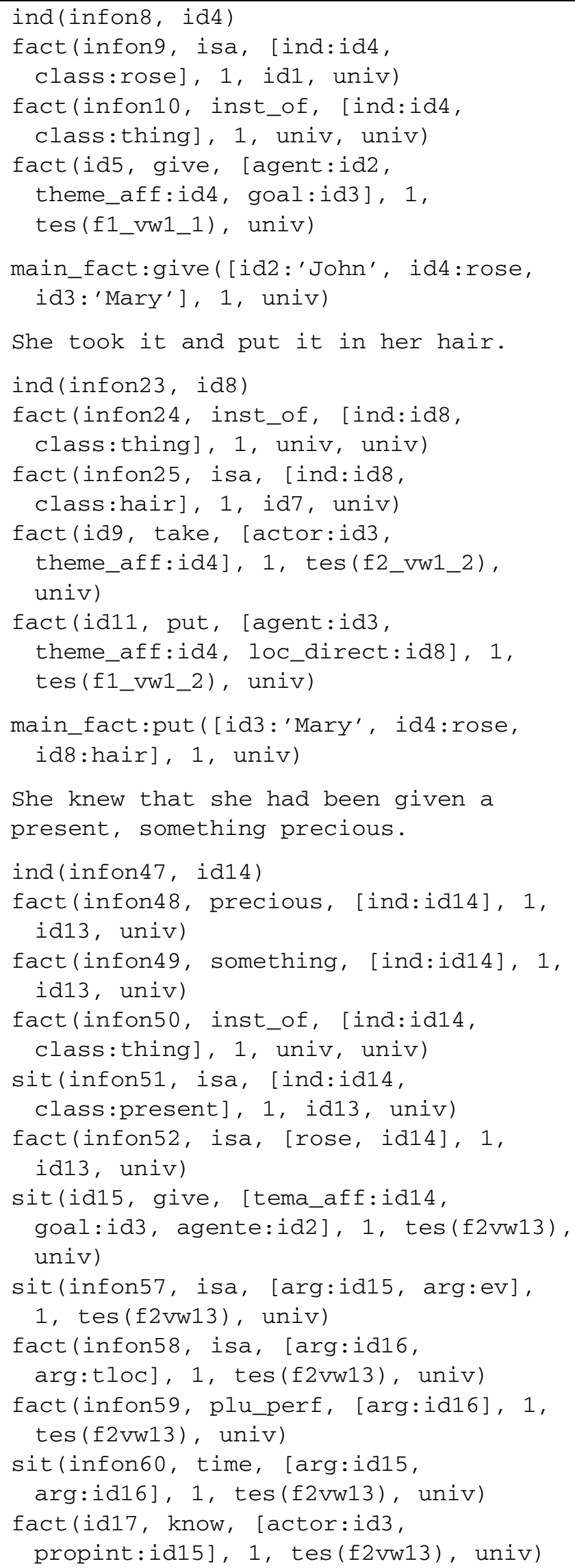


5.2 Examples of pragmatically and semantically motivated IEEs

Text 3. A bus came round the corner. I signalled to the driver to stop.

- Bridging coreference between THE DRIVER and A BUS

Text 4. Lo nominarono delegato, cioè una specie di funzionario viaggiante. Questo era un compito che gli calzava a pennello/They appointed him delegate, i.e. a kind of travelling officer. This was a task which fitted well with his inclination.

- Indefinite eventive NP to cospecify an eventive property in the previous adjacent proposition, by means of the deictic pronoun QUESTO/This

\subsection{Pragmatically motivated IEEs}

Text 5. Mary picked up the phone and called Jason. Her husband, she thought, would have considered such a move as untruthful and utterly base.

Triggering LD: Indefinite NP headed by a deictic modifier SUCH

- Move classified as [activity,eventive] nominal

Computational Strategy: Recover coreference with previous adjacent events.

Mary picked up the phone and called Jason.

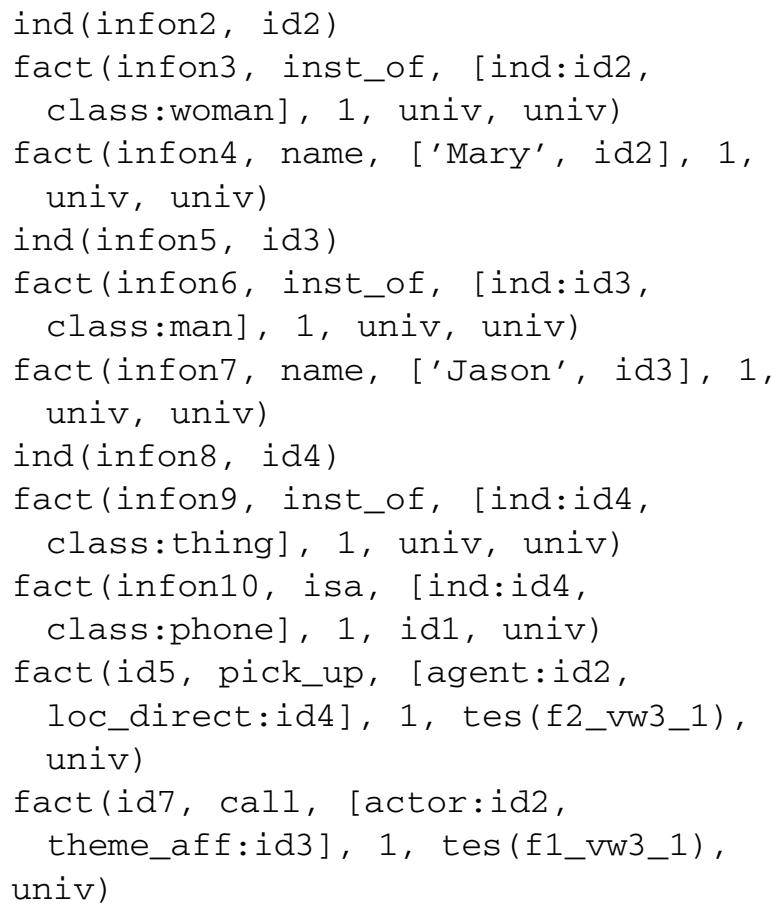

Her husband, she thought, would have considered such a move as untruthful and base.

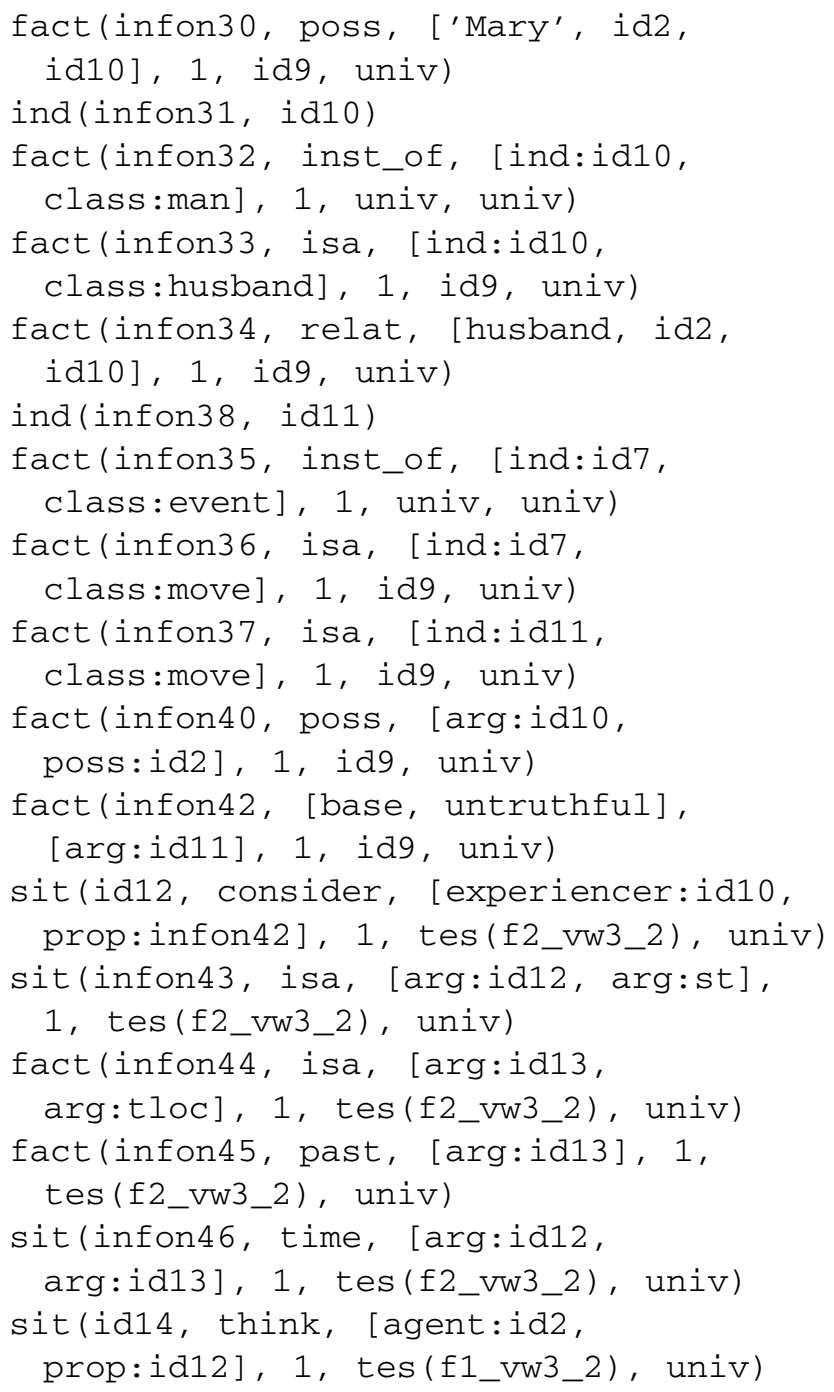

Text 6. John said to himself, "Sara will marry that man", without any resentment. Richard would marry Sara. He felt strongly about that.

Triggering LD: Text expresses the protagonist's point of view

Computational Strategy: John is asserted Subject of Consciousness and Controls a Domain of Point of View. HE is made to corefer with JOHN, because any intervening material is computed as being asserted from JOHN's point of view.

Richard would marry Sara. He felt strongly about that.

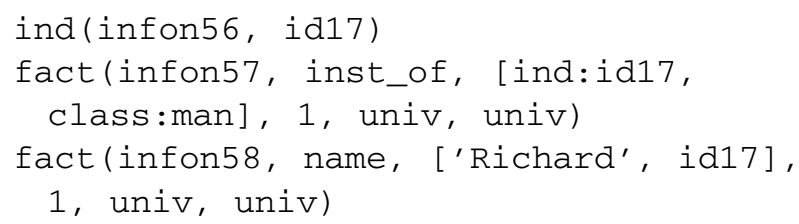




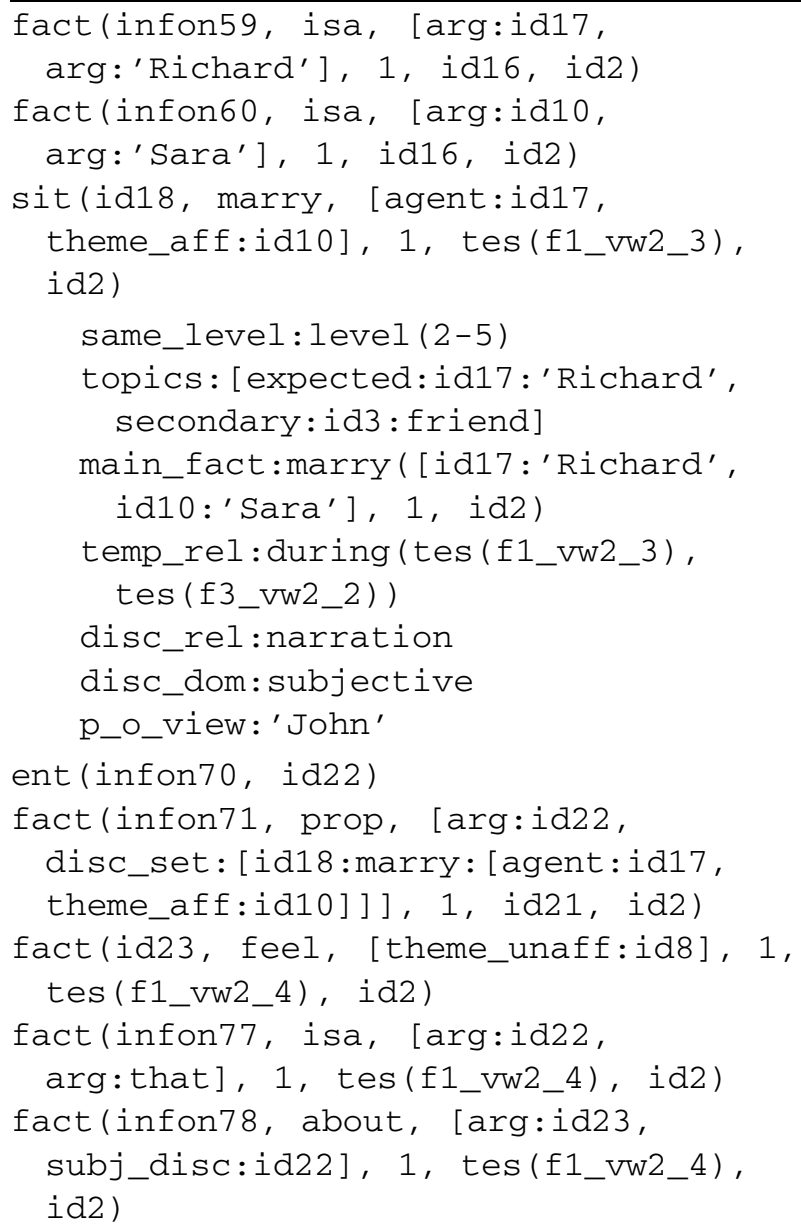

\section{Implicit entities and implicatures}

Conversational implicatures and implications in general, are based on an assumption by the addressee that the speaker is obeying the conversational maxims (Grice 1975), in particular the cooperative principle. The well-known example from Levinson $(1983,107)$,

Text 7.

A: Can you tell me the time?

B: Well, the milkman has come.

requires that both interlocutors share the same spatiotemporal location, besides the same conventions and habits. Not everywhere can you find milkmen go around delivering milk.

Now consider the following example again from Levinson (1983, p. 104).

Text 8.

A: I've just run out of petrol.

B: Oh; there's a garage just around the corner.

Here we see that spatiotemporal locations are even more important: if speaker A needs fuel then the addressee indicates a spatial location, the garage, which in addition has to be open-hence a temporal location. More on this example below.

So, we would like to regard the mechanism that recovers standard implicatures and conversational implications in general, as a reasoning process that uses the knowledge contained in the semantic relations actually expressed in the utterance to recover hidden or implied relations or events as we call them. This reasoning process can be partially regarded as a subproduct of an inferential process that takes spatiotemporal locations as the main component and is triggered by the need to search for coreferent or cospecifiers to a current definite or indefinite NP head. This could be interpreted as bridging referential expression entertaining some semantic relation with previously mentioned entities. In Text (8) the initial inference would be triggered by the metonymy relation intervening between "petrol" and CAR. At the same time CAR would be the trigger of the GARAGE reference, always metonymic. If we consider now Text (7), we see that the request of the current time is itself bound to a spatiotemporal location. Using the MILKMAN rather than a WATCH to answer the question, is relatable to spatiotemporal triggers. In fact, in order to infer the right approximate time, we need to situate the COMING event of the milkman in time, given a certain spatial location. Thus, it is just the "pragmatic restriction" associated with SPACE and TIME that is implied in the answer, that may trigger the inference. More on this topic below.

\subsection{The restaurant text}

To exemplify some of the issues presented above we present a text by Sanford and Garrod (1998), debated in Anderson et al. (1983), QJEP (1983) called the Restaurant text. In this text, entities may be "scenario-dependent" (Sanford and Garrod 1981) or main characters that are independent thereof. While the authors use the text for psychological experimental reasons, we will focus on its computability. So first of all the sentences making up the text, here below,

Text $9 .^{1}$

0 . At the restaurant.

1. John went into a restaurant.

2. There was a table in the corner.

3 . The waiter took the order.

4. The atmosphere was warm and friendly.

5. He began to read his book.

Here below we will only comment on implicatures and implicit arguments. The text is also defined a "psychological statement" text, i.e. it includes sentence (4) that represents a

\footnotetext{
${ }^{1}$ The text has also been used in the challenge of the Shared Task associated to STEP2008, and its full analysis is available at the link with the same name under SIGSEM main page.
} 
psychological statement, that is it expresses the feelings and is viewed from the point of view of one of the characters in the story. The relevance of the sentence is its role in the assignment of the antecedent to the pronominal expressions contained in the following sentence. Without such a sentence the anaphora resolution module would have no way of computing "John" as the legitimate antecedent of "He/his". However, in order to capture such information, a system has to compute Point of View and Discourse Domain on the basis of Informational Structure and Focus Topic by means of a Topic Hierarchy algorithm based on Grosz (1981), Sidner (1983), and Grosz and Sidner (1986), which has been lately evaluated in Delmonte (2007-2009).

\subsection{Subjectivity and point of view hide IEEs}

Another important category of IEEs is constituted by what we called the Subject of Point of View, which is usually coincident with the narrator or the author in newswire texts; except for those utterances which report directly or indirectly what some character in the narrated events has said. It goes without saying that in each case it is the point of view of the speaker/narrator that is being reported. There is an additional important case that has been brought to the attention of computational linguistics lately_-but had already been there for some time: and it is Subjectivity and Subject of Consciousness (hence SOC). Whenever a text uses evaluative statements by means of emotional and subjective markers like adjectives and adverbials that contain semantic evaluation, it may be the case that it is not the narrator's point of view that is being represented but some other character. Now consider our small text: it contains a "psychological statement" (see Sanford and Garrod 1988) sentence, where we realize that the scene is viewed from the perspective or the point of view of one of the participants which is not the narrator. In our case, it is JOHN's point of view and not the WAITER's that needs to be stated. This might or might not be of interest to the semantic representation of the text. In fact it is of paramount importance because it impinges on the decisions that the anaphoric resolution component has to take. The following sentence contains two pronouns which need to be bound at discourse level by a suitable antecedent.

GETARUNS system has a way for coping with this problem that requires setting up a stack with the current SOC. This is done by means of the module called Semantic Informational Structure which is responsible for deciding whether an utterance is Subjective or Objective, using a number of propositional level attributes (see Delmonte 2007-2009). Eventually, the SUBJect of the Subjective utterance will be asserted as the current SOC; or in case that is not suitable - it must be an entity marked as "human"- the Focus of Discourse is chosen. Usually SOCs require a presubjective clause to be present and has scope at the level of
Domain of Point of View, which may span a certain number of clauses until an Objective utterance is reached. Now consider that "John" is computed as the Main Topic or Focus of discourse in Sentence (2) thanks to the inference drawn by the semantic component that takes the "table" as a standard implicature and looks for other possible related events. When the "waiter" appears on the scene as the SUBJect of a "take_order" event, it is again understood as common knowledge in the current scenario, basically thanks to the use of a definite NP. Thus bridging reference resolution is activated thanks to inference drawn by the system on the basis of WordNet. This prevents the Topic Hierarchy algorithm from discarding "John" from the topic stack or treat it as "potential" topic, a slot where no (longer) relevant entities are assigned. Conversely, the system computes John as "secondary" and asserts the "waiter" as a new "expected" topic. The evolution of Topic and SOC stack is shown here below: we show the Weighted List of Topics containing all referring expressions with their attributes as they have been computed from the f-structure. We list below the Rhethorical Structure where we have a state for each sentence, and a list of topics, this time with their semantic identifier. For sentence 4 we also show the Semantic Informational Structure, where we compute propositional level attributes for each clause. This is where the SOC may ensue and may be used to influence the behaviour of the referential system in the following discourse.

\section{Sentence 1 .}

Centering and Topic Hierarchy WEIGHTED LIST OF TOPICS:

ref_ex(sn4, John, [+ref, def0, nil, nil, -pro, -ana, -class], 3, mas, sing, [human], subj/agent)/15

ref_ex(sn5, restaurant, [+ref, -def, nil, nil, -pro, -ana, +class],

3, nil, sing, [place], obl/locat)/29

'RHETORICAL STRUCTURE:'

state $(1$, change)

topic(1, expected, John, id4, sn4)

topic(1, potential, restaurant, id1, sn5)

Sentence 2.

Centering and Topic Hierarchy

WEIGHTED LIST OF TOPICS:

ref_ex(sn5, table, [+ref, -def, nil, nil, -pro, -ana, +class], 3,

nil, sing, [place, object], subj/theme_bound)/46

ref_ex(sn6, corner, [+ref, +def, nil, nil, -pro, -ana, +class],

3, nil, sing, [place, object], ogg/nil)/146

\section{RHETORICAL STRUCTURE:}

state $(2$, continue)

topic(2, main, John, id4, nil)

topic (2, potential, table, id7, sn6)

ent (infon34, id12)

fact (infon35, inst_of, [ind:id12,

class:place], 1, univ, univ) 


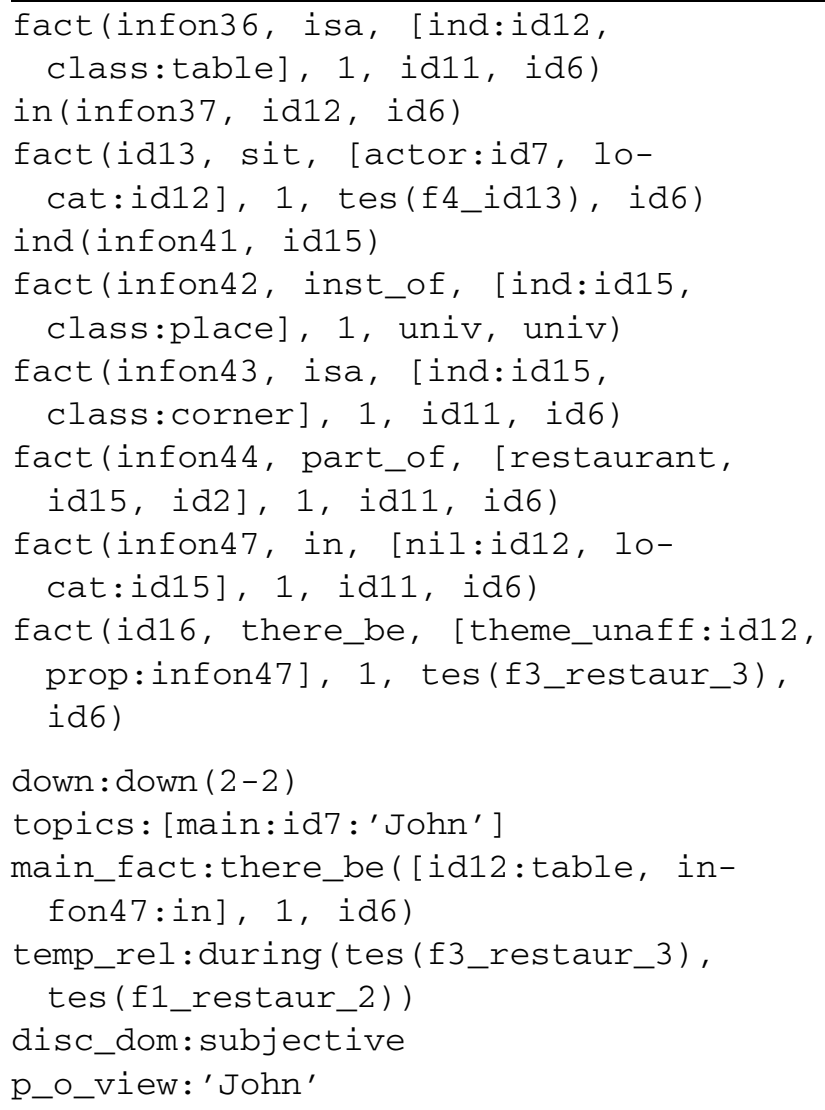

Sentence 3.

Centering and Topic Hierarchy

WEIGHTED LIST OF TOPICS:

ref_ex(sn3, waiter, [+ref, +def, nil, nil, -pro, -ana, +class], 3, mas, sing, [human, social], subj/agent)/15

ref_ex(sn5, exist, [+ref, -def, +part, nil, -pro, -ana, +me], nil, nil, nil, [human, animate], obj2/goal)/217

\section{RHETORICAL STRUCTURE:}

state(3, retaining)

topic(3, expected, waiter, id15, sn3)

topic(3, secondary, John, id4, sn5)

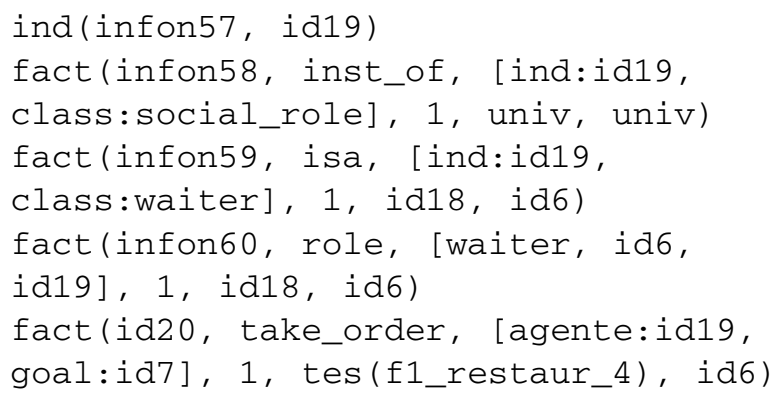

Sentence 4.

Centering and Topic Hierarchy

WEIGHTED LIST OF TOPICS:

ref_ex(sn5, atmosphere, [+ref, +def, nil, nil, -pro, -ana, +class], 3, nil, sing, [substance, abstract], subj/theme_bound)/119
RHETORICAL STRUCTURE:

state $(4$, continue)

topic(4, main, John, id4, nil)

topic(4, potential, atmosphere, id19, sn5)

Semantic Informational Structure

CLAUSE IDENTIFIER: 4-n1

CLAUSE TYPE: main/prop

FACTUALITY: factive

CHANGE IN THE WORLD: null

RELEVANCE: background

TEMP. REL.: during(tes(f1_t34), tes(f1_t33))

DISCOURSE FOCUS: tes(f1_t34)

DISCOURSE RELATION: explanation

DISCOURSE DOMAIN: subjective SUBJECT OF CONSCIOUSNESS: John

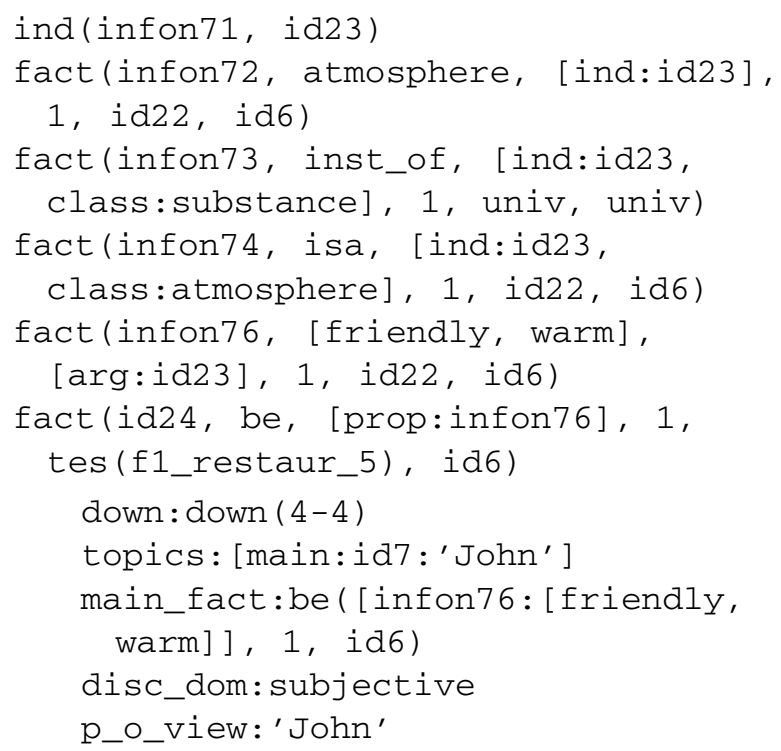

By asserting the SOC, the system is now ready to bind possible external pronouns to it, provided nothing-like feature mismatch-prevents it. And this is what happens in the following sentence, as shown here below, where we see that the pronoun "he" is eventually bound to the SOC.

\section{Sentence 5 .}

Centering and Topic Hierarchy

WEIGHTED LIST OF TOPICS:

ref_ex(sn4, he, [+ref, +def, nil, nil, +pro, +ana, +me], 3,

mas, sing, [human], subj/actor)/ -14

ref_ex(sn5, book, [+ref, +def, nil, nil, -pro, -ana, +class], 3, nil, sing, [object, inform], obj/theme_aff)/36

\section{RHETORICAL STRUCTURE:}

state $(5$, continue)

topic (5, main, John, id4, sn4)

topic(5, secondary, atmosphere, id19, nil)

topic (5, potential, book, id23, sn5) 
The Discourse Model records the output of the anaphora resolution module which searches for SOCs before looking into the Topic Stack.

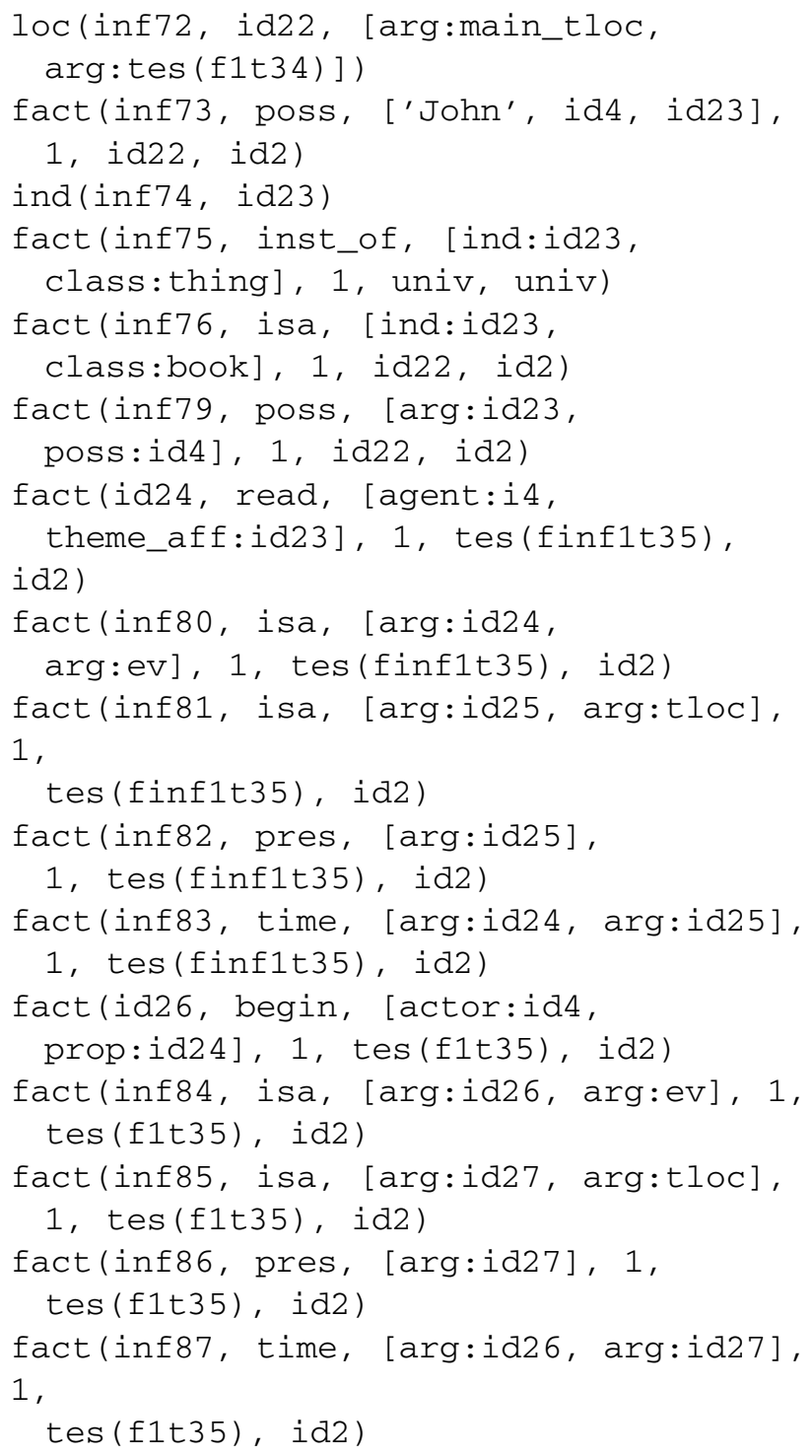

\section{Commonsense reasoning and IEEs}

We will concentrate our attention to sentence (3) at first, which is an example of INI. To account for the fact that whenever a waiter takes an order there is always someone that makes the order, we compute TAKE_ORDER as a compound verb with an optional GOAL argument that is the person ORDERing something. The system then looks for the current Main Topic of discourse or the Focus as computed by the Topic Hierarchy Algorithm, and associates the semantic identifier to the IEE. This latter procedure is triggered by the "existential" dummy quantifier associated with the implicit optional argument. However, another important process has been activated automatically by the presence of a singular definite NP, "the WAITER", which is searched at first in the Discourse Model of entities and properties asserted for the previous stretch of text. Failure in equality matching activates the bridging mechanism for inferences which succeeds in identifying the WAITER as a Social Role in a Restaurant, the current Main Location. In fact, "the waiter" might have been bound to JOHN if the Main Location mechanism were not present in the system.

Consider now sentence (2) which introduces a TABLE as main Topic. This type of sentence is called "presentational" and has the pragmatic role of "presenting" an entity on the scene of the narration in an abrupt manner, or as Centering would define it with a SHIFT move. However, the TABLE does not constitute a suitable entity to be presented on the scene and the underlying import is triggering the inference that "someone is SITting at a TABLE". This inference is guided by the spatiotemporal component of the system. GETARUNS is equipped with a spatiotemporal inferential module that asserts Main SpatioTemporal Locations to anchor events and facts expressed by situational infons. This happens whenever an explicit lexical location is present in the text. In our case, the location expressed is the Restaurant. This can either be part of the title or just be derived from the first sentence of the text, where it has the role of LOCATion argument of the governing verb GO and the preposition INTO. The second sentence contains an expressed location: the CORNER. Now, the inferential system will try to establish whether the new location is either a deictic version of the Main Location; either it is semantically included in the Main Location, or else it is a new unconnected location that substitutes the previous one. The "corner" is in a meronymic semantic relation with "restaurant" and thus it is understood as being a part_of it. This inference is the trigger of the IMPLICATURE that the TABLE is a metonymy for the SITting event. Consequently, when the system tries to corefer, cospecify or assert new semantic individuals, it will find an Indefinite expression "a table" which will not just constitute literally that the text presents a new entity TABLE, but that the IE is involved with a related event. The Entity implied is again understood as the Main Topic of current Topic Hierarchy, i.e. JOHN.

The procedure invoked by the system to produce such an implicature is this:

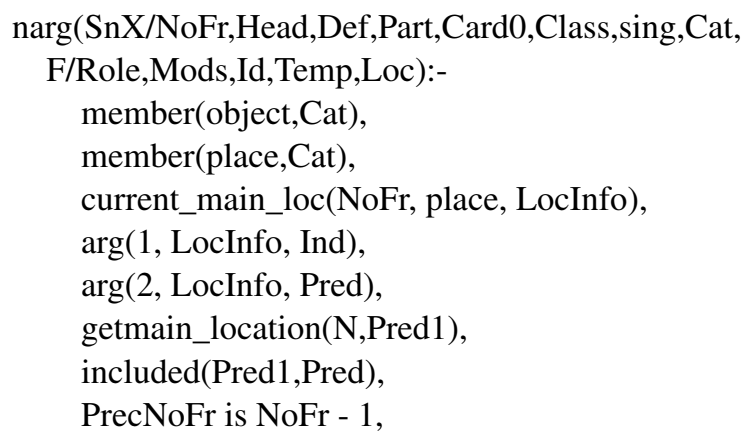


topic(PrecNoFr,main,Idy),

ind(_,Idy),

find_card(SnX,Num,Part, Quant, Card0,Card), creater(NoFr,ind,SnX,Head,Card,Quant,Num,Id, Temp, Loc),

class_props(NoFr, SnX, Cat, Id, fact, isa, Head, 1, Temp, Loc),

assert1(NoFr,SnX,in(Id,Ind)), create_infer_rel(NoFr, Pred1, Idy,Id,Temp, Loc), !.

where included $(\mathrm{X}, \mathrm{Y})$ is a call to WordNet that checks all possible inclusion relations: hyponymy, hyperonymy, meronymy, and so on. The call "current_main_loc" is specified for spatial "place" locations and recovers the Predicate the "restaurant". Then the semantic index of the Main Topic is searched-Idy - and passed down to the predicate that will compute the implicature. The following proceduresfind_card, creater, class_prop, assert1—produce the other semantic index associated to TABLE and assert its semantic properties; "assert1" asserts an inclusion for the location Table into the Main location.

Then there is the final call "create_infer_rel" which has the task of searching for unexpressed relations intervening in the current spatiotemporal location. Then we look for current main location specified for spatial "place" locations and recover the Predicate, the "restaurant". Then the semantic index-Idy — of the Main Topic is searched and passed down to the predicate that will compute the implicature. The following procedures produce the other semantic index associated to TABLE and assert its semantic properties; it asserts an inclusion for the location Table into the Main location.

Then there is the final call which has the task of searching for unexpressed relations intervening in the current spatiotemporal location. To solve this problem in a principled manner we needed commonsense knowledge organized in a computationally tractable way. This is what CONCEPTNET 2.1 (Liu and Singh 2004) actually constitutes. ConceptNetavailable at www.conceptnet.org - is the largest freely available, machine-useable commonsense resource. Organized as a network of semi-structured natural language fragments, ConceptNet consists of over 250,000 elements of commonsense knowledge. At present there are 19 semantic relations used in ConceptNet, representing categories of, inter alia, temporal, spatial, causal, and functional knowledge. The representation chosen is semi-structured natural language using lemmata rather than inflected words. The way in which concepts are related brings to mind "scripts", where events may be decomposed in Preconditions, Subevents and so on, and has been inspired by Cyc (Lenat 1995).

ConceptNet can be accessed in different ways, we wanted a strongly constrained one. We chose a list of functions that encode pieces of knowledge and use those functions together with the information available at a certain point of the computation to derive Implicit Information. In other words, we assume that what is actually being said hides additional information which, however, is only implicitly hinted at. What we need is a predicate constrained by a conceptual function and other predicates. So first of all the list of functions,

allsceneryevents(['SubEventOf','FirstSubeventOf', 'DesiresEvent','Do',CapableOf', 'FunctionOf','UsedFor',

'EventRequiresObject',LocationOf']

Then the call that searches ConceptNet for implicit information,

create_infer_rel(NoFr, MainLoc, AgentId, CurrLocatId,

Temp, Loc):-

allsceneryevents(CondEvents), member(Type, CondEvents),

MatchScenery=..[Type, [go, Prep, MainLoc], [Event, Preps, CurrLocat])

...

Infon=..[fact, EvId, Event, [actor:AgentId, locat:

CurrLocatId], 1, tes(Tr3), Loc], assert(Infon) ...

If the call is successful, we end up by recovering a predicate SIT in the slot Event, and use this predicate to assert an additional property associated to the Topic of discourse. So eventually, the system checks for implicatures because it is triggered by the unsuitability of the current entity-the TABLE-as topic of discourse.

With a similar strategy the non-standard implicatures in Text 8 can be resolved. We reproduce it here below.

Text 8 .

A: I've just run out of petrol.

B: Oh; there's a garage just around the corner.

There are a number of missing conceptual links that need to be inferred, as follows:

\section{Infl: the CAR has run out of petrol \\ Inf2: the CAR NEEDS petrol \\ Inf3: garages SELL PETROL for cars}

In addition, in order to use ConceptNet we need to translate "petrol" and "garage" into "gas/gasoline" and "gas station" respectively. This passage is not just a mere translation but requires contextual information to tell apart the two meanings associated to the word "garage"- that is the place where you keep your car, and the place where you get gas. Now we can query the ontology as we did previously and will recover the following facts. The whole process starts from the first utterance and uses RUN OUT OF GAS,

(Do "car" "run out of gas")

Then we can use GAS STATION and CAR to build another query and get, 
(Do "car" "get fuel at gas station")

where FUEL and GASoline are in IsA relation. We may still get additional information on the reason why this has to be done,

(Do "person" "don't want to run out of gas")

(SubeventOf "drive car" "you run out of gas")

(Do "car" "need gas petrol in order to function")

(Do "gas station" "sell fuel for automobile")

These may all constitute additional commonsense knowledge that may be used to further explain and clarify the implicature.

A brief comment on Schank's approach (1977)—but see also Mueller (2007) — and the restaurant text. Schank introduces scripts and a theory of conceptual dependencies which are based on primitive actions which are very close to FrameNet's Frames. In Schank's perspective, plans are the means for satisfying goals and they are composed of scripts. To understand a story one needs scripts and a plan. However, unlike what we have done here, scripts are organized with metadata that contain for instance preconditions, instrumental relations etc. and we certainly make no use of preorganized conceptual structures. We do not even make use of Frames, in the literal sense, in order to produce lexical inferences. Everything we do is built by the actual PredicateArgument Structures instantiated in a given text as it is analyzed and represented in situational semantics in a Discourse Model by GETARUNS.

\section{Conclusions}

We have presented a complete treatment of implicit entities and events that encompasses all possible semantically relevant lexically unexpressed elements. This has been implemented in a system called GETARUNS which deals with all these phenomena in a principled way by means of a theoretically validated division of labour between the different modules that make up the whole pipeline. We subdivided IEEs into different categories according to both theoretical and computational criteria. In this way grammatical IEEs are taken care of before lexically semantically motivated ones. In turn these latter come before the need to carry out pronominal binding and anaphora resolution. Finally, when the semantic components are completing their mapping and search the preceding Discourse Model for coreferring/cospecifying entities, procedures that look for implicatures are activated and inferences are fired. This can only be done in presence of a full-fledged semantic interpretation of the current utterance, because it is only by means of its PAS that the appropriate implicit events may be recovered. World knowledge is represented by two repositories: a generic semantic network like WordNet and the commonsense ontology ConceptNet. Again, other similar repositories may be used, but the mechanisms to access them should be the same: no implicature may be recovered without a full semantic interpretation of the triggering utterances. Dialogues and texts are full of IEEs either as elliptic material or as implicated events and we are currently experimenting with Multiparty Meetings Dialogues from ICSI-Berkeley-in order to verify what impact they may have on the overall interpretation process.

\section{References}

Anderson, A., Garrod, S. C., \& Sanford, A. J. (1983). The accessibility of pronominal antecedents as a function of episode shifts in narrative text.

Baker, C. F., Fillmore, C. J., \& Lowe, J. B. (1998). The Berkeley FrameNet project. In Proceedings of COLING-ACL-98, Montreal, Canada.

Bresnan, J. (2000). Lexical-functional syntax. Oxford: Blackwell.

ComLex. http://nlp.cs.nyu.edu/comlex.

Delmonte, R. (2007-2009). Computational linguistic text processing. New York: Nova Science Publishers.

Fellbaum, C. (Ed.) (1998). WordNet: an electronic lexical database. Cambridge: MIT Press.

Grice, H. P. (1975). Logic and conversation. In P. Cole \& J. L. Morgan (Eds.), Syntax and semantics: Vol. 3. Speech acts (pp. 41-58). New York: Academic Press.

Grosz, B. (1981). Focusing and description in natural language dialogues. In A. Joshi, B. Webber, \& I. Sag (Eds.), Elements of discourse understanding. Cambridge: Cambridge UP.

Grosz, B., \& Sidner, C. (1986). Attention, intentions and the structure of discourse. Computational Linguistics, 12, 175-204.

Lenat, D. B. (1995). CYC: a large-scale investment in knowledge infrastructure. Communications of the ACM, 38(11).

Liu, H., \& Singh, P. (2004). ConceptNet: a practical commonsense reasoning toolkit. At http://web.media.mit.edu/ push/ ConceptNet.pdf.

Levinson, S. C. (1983). Pragmatics. Cambridge: Cambridge UP.

Mueller, E. T. (2007). Modelling space and time in narratives about restaurants. Literary and Linguistic Computing, 22(1), 67-84.

The Quarterly Journal of Experimental Psychology Section A (1983). 35(3), 427-440.

Sanford, A. J., \& Garrod, S. C. (1981). Understanding written language: exploration in comprehension beyond the sentence. Chichester: Wiley.

Sanford, A. J., \& Garrod, S. C. (1988). Thematic subjecthood and cognitive constraints on discourse structure. Journal of Pragmatics, 12(5-6), 519-534.

Sanford, A. J., \& Garrod, S. (1998). The role of scenario mapping in text comprehension. Discourse Processes, 26, 159-190.

Schank, R. C., \& Abelson, R. P. (1977). Scripts, plans, goals and understanding: an inquiry into human knowledge structures. Hilsdale: Erlbaum.

Sidner, C. (1983). Focusing in the comprehension of definite anaphora. In M. Brady \& R. Berwick (Eds.), Computational models of discourse (pp. 267-330). Cambridge: MIT Press. 